\title{
The Design and Implementation of Intelligent Greenhouse Monitoring System Based on WEB
}

\author{
Hengguo Zhuang ${ }^{a}$, Ruming Gao ${ }^{b}$, Zhibin $\mathrm{Du}^{\mathrm{c}}$ and Jiansheng Guo ${ }^{\mathrm{d}}$ \\ Beijing CATARC Data \& Technology Center, Tianjin 300000, China \\ azhuanghengguo@catarc.ac.cn, bruminggao1990@163.com, cduzhibin@catarc.ac.cn, \\ dguojiansheng@catarc.ac.cn
}

\begin{abstract}
With the development of Internet and information technology, the traditional greenhouse environmental monitoring system operation is not stable. This paper designed a greenhouse intelligence remote monitoring system based on Web. The system used $\mathrm{B} / \mathrm{S}$ architecture to realize real-time monitoring and adjusting the value of the greenhouse environment factor data. Besides, the system controlled the equipment and collected the data. This monitoring system was stable and strong, and had wide application prospect in the remote data acquisition and transmission, remote monitoring and so on.
\end{abstract}

Keywords: Web; Remote Monitor; Intelligent Greenhouse.

\section{Introduction}

At present, the agricultural of China is in a new stage that transform from traditional style to intensive, high quality and high efficiency. Besides, crop growth is closely related to temperature, humidity, carbon dioxide and light intensity or other environmental factors [1]. Different crops in different growth stages need stay in a suitable development circumstance. With the development of computer network and intelligent automation technology, automatic and precise monitoring of greenhouse environment information has become an important means of facility agriculture. In recent years, the traditional motoring method collected data by the lower computer send data to the host computer through the serial port, then releasing controlling instruction [2]. This kind of monitoring method has many shortcomings, failing to serve multi users or extend.

With the development of computer network and embedded technology, the monitoring technology has been improved rapidly, and the monitoring system has been gradually developed from the centralized monitoring to the remote intelligent monitoring systems. Based on embedded and Web server technology, this paper designs and realizes the intelligent remote monitoring system from the lower computer by the wireless network to the Web server. In the part of lower computer, make the Arduino board as the control core, and add the Ethernet network module, realizing the function of data acquisition and data wireless transmission. In the part of host computer, apply the Java technology under the Eclipse environment, based on the module of design principles and JFinal development framework. Then design the monitoring system of greenhouse through the browser and server $(\mathrm{B} / \mathrm{S})$ mode.

\section{Framework of system}

The system is mainly composed of data acquisition layer, data transmission layer and application layer, as shown in figure 1. Data acquisition layer main role is obtaining the environment factor data through Arduino board and sensors or other hardware equipment. Data transmission layer is mainly sending the environment factors to the web server's IP address and storing them in the database by Arduino Ethernet W5100 network module. The main function of the application layer is to achieve real-time remote data monitoring and control of the implement part based on the framework of JFinal and the information system of host computer combined $\mathrm{B} / \mathrm{s}$ architecture. 


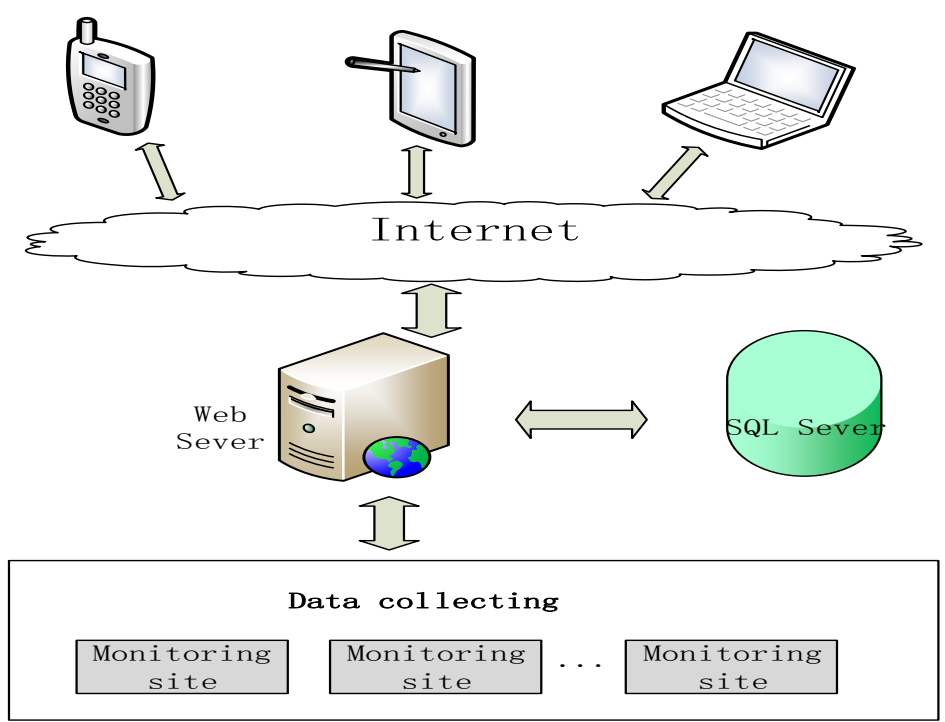

Fig.1 Design of system architecture

\section{The hardware design of system}

\subsection{The hardware design of data acquisition layer}

Data acquisition layer is responsible for the intelligent control of greenhouse environment data and equipment. It is made up of control unit, temperature and humidity sensor, light sensor, carbon dioxide sensor, Ethernet W5000 module and controlling composition. Control unit taking the Arduino Leonardo board as the control core, collects the environment data of illumination, temperature, humidity, carbon dioxide and other factors through sensors, which connects to Ethernet W5000 module by wireless mode. Then using logic circuit control equipment to ventilate and fill light. The overall design of the data acquisition layer is shown in Fig. 2.

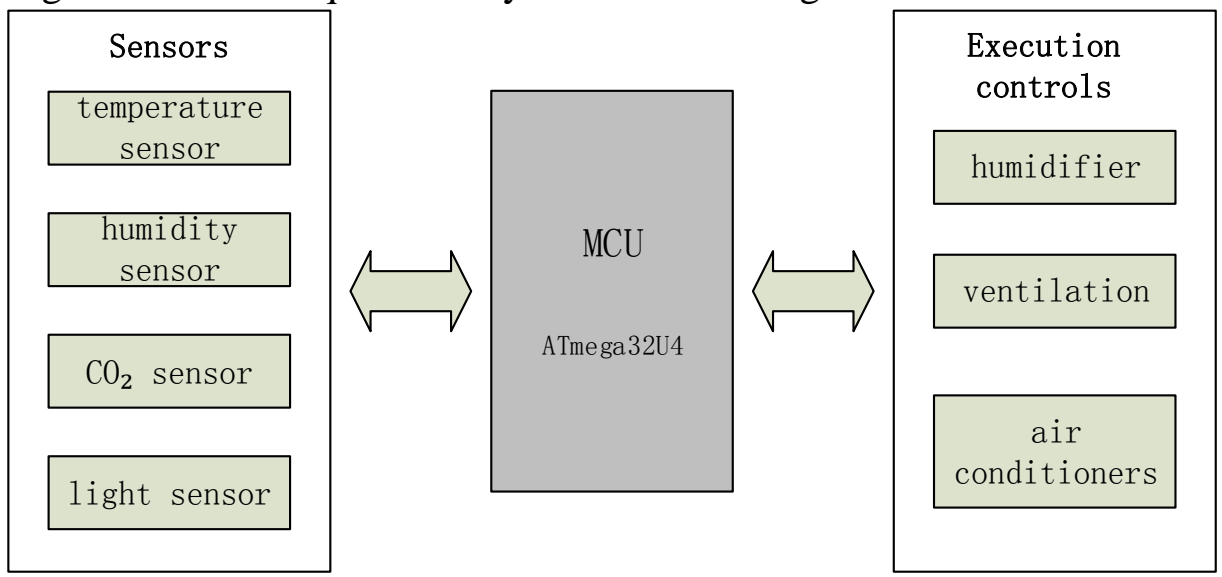

Fig. 2 Design of data acquisition layer

\subsubsection{Circuit of temperature and humidity regulating}

This system regulates the temperature and humidity of the greenhouse in 2 ways. One is through adjusting the roof and the side window, the other is through the air conditioning.

When the temperature or humidity of the greenhouse is too high, it can through the greenhouse monitoring station or directly through the website, remote control the stepper motor to open the window, regulating the temperature and humidity with wind. If the indoor environment can not be kept in the predetermined range with wind, the greenhouse monitoring station start the air conditioner. Through the air regulating temperature and humidity to keep in the range set by the system. Greenhouse monitoring stations achieve the control of air conditioner through the relay, and the circuit is shown in Fig. 3. 


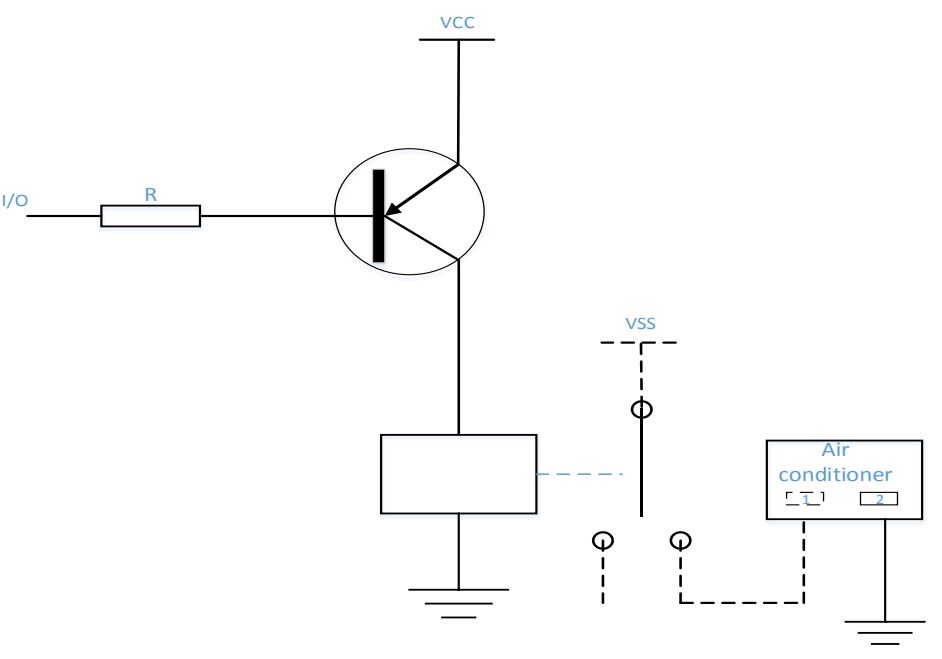

Fig. 3 Circuit of air conditioning control

When the I/O port of microprocessor is at the low-level state, the relay will be charged and feet 1,2 will be connected. At this time, the air conditioner start to work. When the I/O port at high-level state, the relay will be cut down and the air conditioner will be turn off.

\subsection{Design of transmission layer}

The transmission layer is responsible for transferring the collected greenhouse environment data to the specified server. Hardware equipment is mainly composed of Leonardo Arduino control board and Ethernet W5000 network module. After determining their MAC address and IP address, the software uses get or post method to transfer the data to the specified server through wireless mode.

\section{Design of software in the system}

\subsection{Framework of application layer}

The application layer is made up of field control system, database and remote monitoring system. And it is responsible for receiving the collected environment data and storing them in the database. Moreover, the real-time monitoring data curve and environment management data are also kept in the database. According to the design requirements of the remote monitoring system, combining with $\mathrm{B} / \mathrm{S}$ 3-level architecture and JFinal framework in the Eclipse platform, build the system.

\subsection{Design of B/S 3-level architecture}

There are three layers in the B/S 3-level architecture, namely, presentation layer, business logic layer and data access layer.

\subsubsection{Design of presentation layer}

The presentation layer is the first layer of the B/S 3-level providing an interactive interface, which is mainly used to receive the demand of input and output from users and display the results of the Web server implementation. User login interface use the brushless technology of AjAX engine to achieve the partial update page. Besides, real-time monitoring page is an important interface in the presentation layer design. This interface does not need to handle the logic, but send the request of users to the business logic layer. Then the business logic layer access to the database by the data access layer. Finally, results of database show on the real-time monitoring page.

In order to achieve greenhouse environment data curve of real-time dynamic update, use the software of jQuery HighCharts with Ajax engine technology for drawing real-time reporting. jQuery HighCharts is a real-time reporting API, which is user-friendly and displays clearly. Interface of real-time temperature monitoring is shown in Fig. 4. 


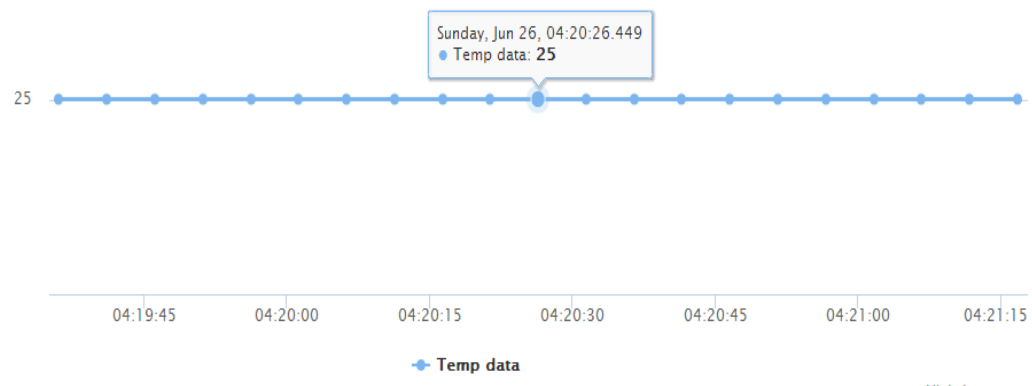

\subsubsection{Design of business logic layer}

Fig.4 Interface of real-time temperature monitoring

The business logic layer is the middle layer of the B/S 3-level layer architecture, which is a bridge connecting the data access layer and the presentation layer. And it mainly realizes the operation of the presentation layer. According to the function of the system, different business components are built, such as user management module, real-time monitoring module, threshold setting module, remote control module, information query module and so on.

\subsubsection{Design of data access layer}

The data access layer is the third layer of the B/S 3-level layer architecture, which provides data services for the presentation layer and the business logic layer. And it mainly realizes the management of the background database. According to the request of the system, build the data management module and data access module. Moreover, based on the JFinal framework, it can be very convenient in the application of the connection and operation of database, through the $\mathrm{C} 3 \mathrm{p} 0$ database connection pool and ActiveRecord database access.

\section{Summary}

After the layout, debugging, and a series of work, the system is in the state of stable operation and reliable performance. It also realizes the automatic acquisition and intelligent control of temperature, humidity, illumination, soil humidity, and $\mathrm{CO} 2$ concentration data. Through the Internet terminal for the remote network monitoring, it improve the intelligent level of greenhouse system and reduce the management cost. The greenhouse management system is promoted in the agriculture science and technology demonstration and it achieves good results.

\section{Conclusion}

Intelligent remote monitoring system adopts many kinds of advanced technology, such as Arduino technology, sensor technology, automatic control technology, computer technology. It designs in modular structure and collects monitoring data through the sensor, controller and wireless network hardware. Besides, it achieves the function of greenhouse environment data real time monitoring, alarm threshold settings, remote control and historical data query.

This paper introduces the main design process of intelligent remote monitoring system. This system can solve the problem of greenhouse environment data monitoring. Users can get the data and control the greenhouse through the browser. This system realizes the intelligent control of the greenhouse and provides a simple, efficient, adaptable design scheme. And the system has wide application prospects in remote data acquisition and transmission, remote monitoring and so on. 


\section{References}

[1] Zhao X C, Li J Q, Liu J, et al. Design and Implementation of Large Computer Room Environment Intelligent Monitoring System Based on Zigbee [J]. Applied Mechanics \& Materials, 2013, 397-400: 1673-1676.

[2] Guan W, Wang C, Cai Y, et al. Design and implementation of wireless monitoring network for temperature-humidity measurement $[\mathrm{J}]$. Journal of Ambient Intelligence \& Humanized Computing, 2015: 1-8.

[3] Zhang, Li; Li, Congcong; Jia, Yushen. Design of Greenhouse Environment Remote Monitoring System Based on Android Platform [C]. International Conference on Applied Engineering and Management, Beijing, SEP 11-14, 2015 2015, 46: 739-744

[4] Al-Aubidy K M, Ali M M, Derbas A M, et al. Real-time monitoring and intelligent control for greenhouses based on wireless sensor network[C]// Multi-Conference on Systems, Signals \& Devices. IEEE, 2014:1-7.

[5] Ming X U, Min Y. Design of Embedded Monitoring System for Greenhouse Based on ARM11 and WinCE [J]. Applied Mechanics \& Materials, 2013, 303-306(3):1477-1480.

[6] Wang W, Cao S. Application Research on Remote Intelligent Monitoring System of Greenhouse Based on ZIGBEE WSN [C]// International Congress on Image and Signal Processing. 2009:1-5.

[7] Dogra A K, Chandra P. DEVELOPMENT OF AN INTELLIGENT CONTROLLER FOR GREENHOUSE MANAGEMENT [J]. International Symposium on Design \& Environmental Control of Tropical \& Subtropical Greenhouses, 2002(578): 377-382. 